\title{
Signal Processing for Distance Measurement Using Laser Voltage Fluctuation Due to Self-Coupling Effect
}

\author{
Takeshi Yoshimatsu, ${ }^{*}$ Norio Tsuda, and Jun Yamada \\ Aichi Institute of Technology, 1247 Yachigusa, Yakusa, Toyota, Aichi 470-0392, Japan
}

(Received March 31, 2017; accepted August 9, 2017)

Keywords: laser distance sensor, self-coupling effect, real-time distance sensing

A self-coupling laser distance sensor detects a distance signal via slight fluctuations in the semiconductor laser terminal voltage due to interference between the laser light and the returned light. The output signal of this sensor is composed of the distance signal, the white Gaussian noise, and a harmonic wave. Conventionally, real-time distance measurement using these signals is impossible. However, although the white Gaussian noise is a random signal, the distance signal is periodic under the condition that the semiconductor laser is driven by a triangular current. In addition, the second-harmonic-wave frequency is twice that of the distance signal frequency. Utilizing these features, we propose a new signal processing technique in which the white Gaussian noise is reduced and the distance signal is corrected on the basis of the second harmonic wave. Hence, real-time distance measurement using a self-coupling distance sensor without a photodiode becomes possible. This processing system is constructed using a system-on-chip device. It is found that one measurement is completed within $2 \mathrm{~ms}$, which is one period of modulated signal, and distances of 10 to $40 \mathrm{~cm}$ can be measured with an average error of approximately $2 \%$.

\section{Introduction}

Production technology development is required in order to realize smart factories. In the future, it is predicted that factory productivity and quality control capability will be considerably improved as factory automation equipment becomes connected to the Internet. Simultaneously, however, innovation regarding industrial sensing systems is required. In particular, the demand for a compact and inexpensive distance sensor is increasing, because the distance sensor is a frequently used industrial sensor.

The light intensity of a laser diode (LD) fluctuates slightly when some of the light scattered from the target surface interferes with the laser light within the LD active layer. This phenomenon is called the "self-coupling effect." A self-coupling laser sensor can utilize this effect for measurement, e.g., to measure distance, ${ }^{(1-3)}$ microdisplacement, ${ }^{(4-6)}$ movement speed, ${ }^{(7,8)}$ and sound waves. ${ }^{(9,10)}$ In addition, the self-coupling effect induces slight fluctuations in the laser oscillation wavelength and terminal voltage. ${ }^{(11,12)}$ Therefore, if the terminal voltage fluctuation is utilized instead of a photodetector, this sensor can act as a compact and inexpensive photodetectorfree sensor. Self-coupling laser distance sensors using terminal voltage fluctuation have various applications, e.g., for the position control of robot arms, as collision avoidance sensors, and for shape measurement.

${ }^{*}$ Corresponding author: e-mail: w15804ww@aitech.ac.jp

http://dx.doi.org/10.18494/SAM.2017.1609 
The distance signal detected by the self-coupling laser distance sensor is called a mode hop pulse (MHP). The MHP, which can be detected from the laser terminal voltage fluctuation, overlaps the white Gaussian noise (WGN); thus, only the fast Fourier transform (FFT) is used for signal processing involving MHP. However, use of the FFT requires the execution of an enormous amount of calculation processing and, therefore, expensive equipment is necessary for fast signal processing. Furthermore, real-time measurement using MHP in an industrial distance sensor is difficult. Therefore, research on inexpensive high-speed signal processing techniques other than the FFT has been conducted. The signal counting correction method is a statistical signal processing technique proposed as an alternative to the $\mathrm{FFT}^{(13)}$ which is based on the statistical features of the binarized MHP and binarized noise. In a previous paper, statistical signal processing based on the signal counting correction method was performed. ${ }^{(14,15)}$ In that study, to obtain the statistical features of the binarized signal measurement, processing of the binarized-signal pulse width was required. However, in the case of strong binarized noise, such that the MHP pulse width is immeasurable, the signal counting correction method cannot be implemented. In addition, if the scattering light intensity is strong, a signal with twice the frequency of MHP is imprinted in the output. This signal is the self-coupling signal imprinted in the output by the second harmonic wave, which occurs as a result of the irradiation of laser light on the object targeted for measurement. ${ }^{(16)}$ The signal counting correction method does not assume the existence of this harmonic wave. Thus, the measurable range is limited, and the measurement time is longer than one period of the modulated signal.

However, under the conditions that the LD is driven by a triangular current and the target does not move, the MHP occurs at a constant period, as the LD wavelength varies linearly. Therefore, in this study, we propose a technique that allows reduced processing of the WGN for the first time, by utilizing the MHP periodicity and the statistical processing of the self-coupling signal generated by the harmonic wave. To perform both reduction processing of the binarized noise and the numerical calculation for the statistical processing, Zybo, a system-on-chip (SoC) device, is used. Thus, we propose a new signal processing method and successfully measure distance via real-time processing of the MHP obtained via the LD terminal voltage fluctuation.

\section{Measurement Principle}

\subsection{Mode hop pulse}

A detailed explanation of the self-coupling effect was provided previously. ${ }^{(3)}$ Therefore, this effect is summarized only briefly in this paper. In the same longitudinal mode, the LD oscillation wavelength changes in proportion to the applied voltage. In addition, when the product of half the wavelength and some integer is equal to the measurement distance, the maximum fluctuation in the applied voltage due to the self-coupling effect is obtained. If the voltage applied to the LD is triangularly modulated, periodic voltage fluctuation is caused by the self-coupling effect in one period of the modulated signal. Periodic voltage fluctuation due to the self-coupling effect is called MHP, and the MHP frequency $F$ is given by

$$
F=4 I_{m} f_{m}\left(1 / \lambda^{2}\right)(d \lambda / d I) L
$$

where $L$ is the distance to the target, $I_{m}$ is the modulated current amplitude, $f_{m}$ is the modulated 
frequency, $\lambda$ is the laser oscillation wavelength, and $d \lambda / d I$ is the modulation efficiency. The distance resolution is the length at which the MHP changes by one. Note that the LD coherence length restricts the measurable distance. The output waveform obtained from the LD terminal voltage and the FFT analysis result are shown in Figs. 1(a) and 1(b), respectively. To detect the MHP only, the triangular voltage applied to the LD is removed. This distance sensor output has considerable WGN. If the intensity of the returned light noise is high, a second harmonic wave is imprinted in the output. ${ }^{(16)}$

\subsection{Problem of signal binarization for wide-range measurement}

In this digital signal processing technique, the width of the pulse generated within the processing time is measured sequentially, and a histogram is created in order to calculate the MHP intensity. To prevent changes in the logic voltage level in response to minute noise, the output is binarized using a hysteresis comparator, where $+V_{\text {th }}$ and $-V_{\text {th }}$ are its threshold values [Fig. 2(a)]. The intensity of MHP decreases with increased measurement distance. Designing the threshold value with almost half the amplitude is important for high-speed and wide-range measurement. However, the problem of the considerable WGN cannot be ignored. A broadband band-pass

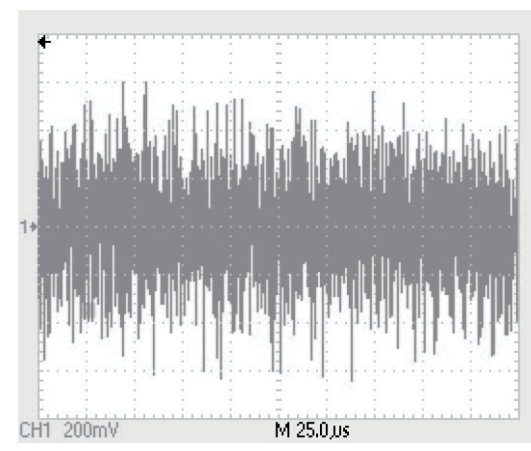

(a)

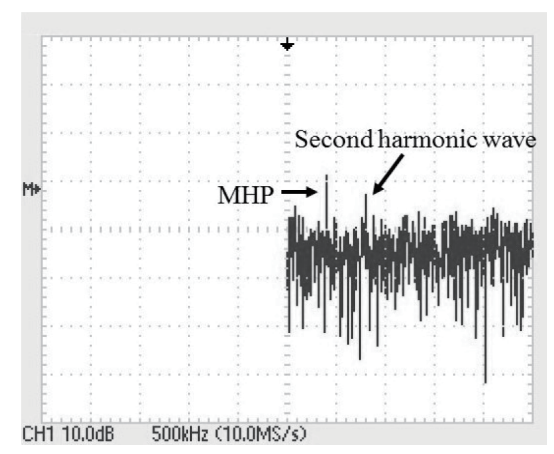

(b)

Fig. 1. MHP measurement results. (a) MHP signal from LD terminal voltage. (b) FFT analysis result of LD terminal voltage.

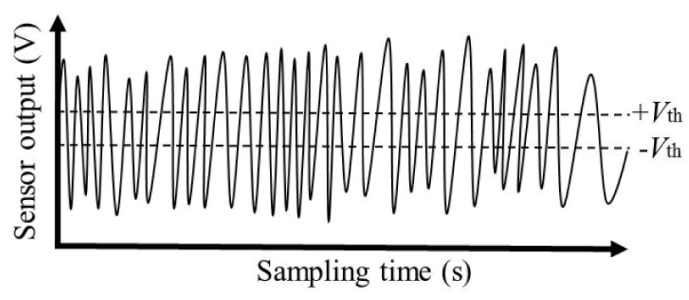

(a)

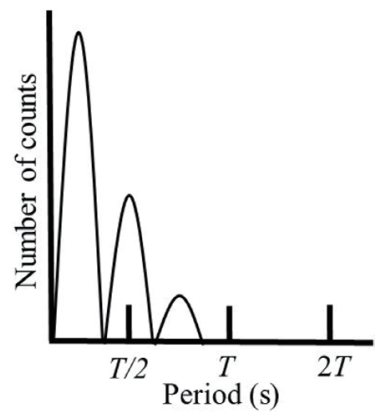

(b)

Fig. 2. (a) Schematic of terminal-voltage waveform and comparator threshold values. (b) Histogram of binarized MHP period. 
filter (BPF) with an amplifier is designed in the receiver circuit of this sensor. Because the MHP voltage obtained from the LD terminal voltage fluctuation is less than $0.1 \mathrm{mV}$, significant gain amplification is necessary. However, the WGN in the bandwidth is also amplified here.

When the threshold is closer to half the amplitude, the WGN has a greater influence. In this case, the obtained pulse width is shorter than half the MHP period. Thus, a histogram such as that shown in Fig. 2(b) is obtained. Here, $T$ is the MHP period for distance data. Because a lot of binarized WGN is mixed into the binarized MHP, MHPs are not measured at all; thus, $T$ and the statistical feature of the $\mathrm{MHP}^{(13)}$ are not obtained.

In addition, when the measured distance is closer, a second harmonic wave is usually imprinted in the output, resulting from interference between the laser light and the high-intensity returned light. The second harmonic frequency is $2 F$. Even if the MHP is measurable, the number of harmonic wave counts is greater than or equal to the number of MHP counts. Thus, the incorrect signal may be selected for processing. To solve these problems, reduction processing of the WGN and correction processing of the second harmonic wave are necessary.

\subsection{Noise reduction processing}

When the threshold is approximately half the amplitude of the sensor output, it is impossible to distinguish between the MHP, WGN, and the harmonic wave in the binarized signal. On the other hand, if the target object does not move, all the pulse widths of the MHP superposed on the triangular wave are equal. In addition, the WGN pulse width is random. Therefore, a noise reduction circuit using the above two features is designed. Figure 3 shows a schematic diagram

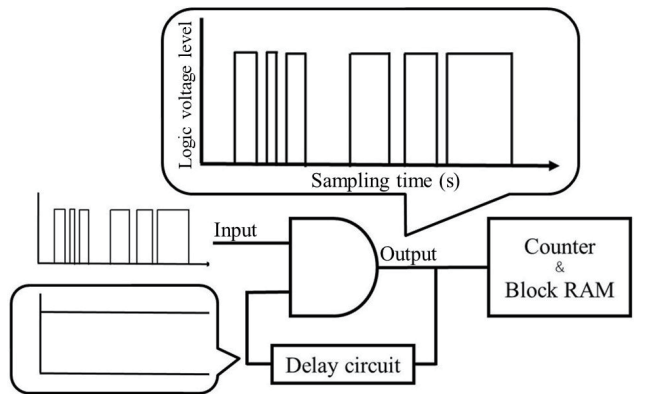

(a)

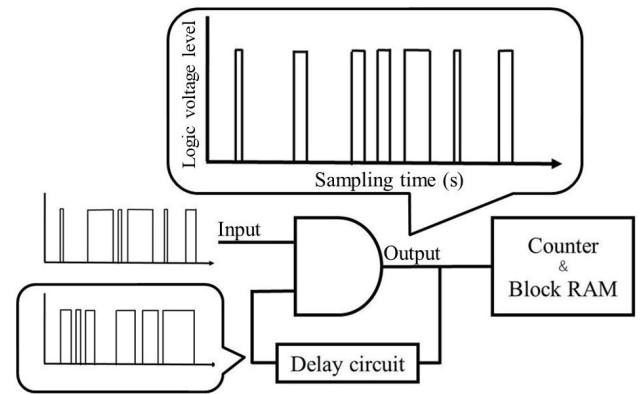

(b)

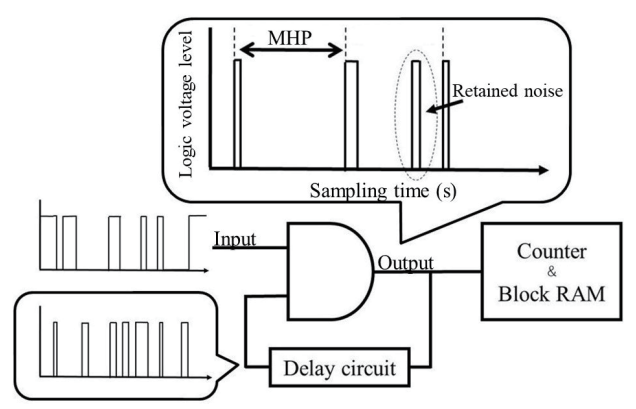

(c)

Fig. 3. Schematics of noise reduction process. (a) Binarized signal immediately after circuit driving. (b) Logical product of binarized input signal and delay circuit output. (c) Logical product of binarized input signal and binarized signal with reduced noise. 
of the noise reduction circuit and the reduction processing flow. The MHP occurs periodically, because the LD wavelength changes linearly as a result of the triangular input current. On the other hand, the WGN is a random signal. Therefore, after the increasing or decreasing interval of the triangular signal is divided, the MHP is detected via the repeated calculation of the logical product.

First, as shown in Fig. 3(a), the logical product of the binarized high-level signal of the delay circuit and the binarized input signal into the device is calculated, and the output signal is input to the delay circuit. Next [Fig. 3(b)], the logical product of the binarized signal within the delay circuit and the newly binarized input signal into the device is calculated, and the output signal is again input to the delay circuit. This logical product calculation is repeated. The logical noise level becomes low and the logical product is not obtained, because the WGN pulse width continues to change at random. However, because all the MHP pulse widths are equal, the logical product of the MHP of the binarized input signal and the MHP stored in the delay circuit is easily calculated. By repeating this processing, the binarized WGN is reduced and the binarized MHP is obtained, as shown in Fig. 3(c). If all the internal bits of the delay circuit are of low level, all bits are inverted. The processing step returns from that in Fig. 3(c) to that in Fig. 3(a). As the delay time is shorter, the processing speed is higher. However, the delay circuit cannot store a long-period MHP. In addition, because the MHP is superposed on the modulated signal, the delay time must be determined based on one period of the modulated signal. Specifically, the delay time must satisfy

$$
T_{d e l}=\left(T_{m} / 4\right) n,
$$

where $T_{d e l}$ is the delay time, $T_{m}$ is the measurement signal period, and $n$ is an integer. The larger the value of $n$, the faster the processing speed.

\subsection{Signal correction based on second harmonic wave}

Increased processing time is required to reduce the second harmonic wave, because this wave is not a random signal. Figure 4(a) is a schematic of the histogram for close-range measurement before signal correction. When the returned-light intensity is high, the second harmonic wave is imprinted in the output. The second-harmonic-wave intensity is weaker than the MHP intensity.

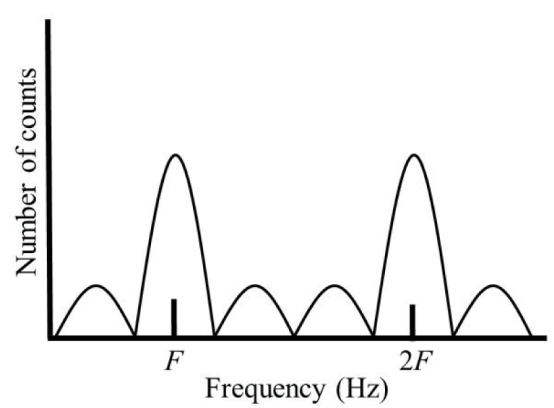

(a)

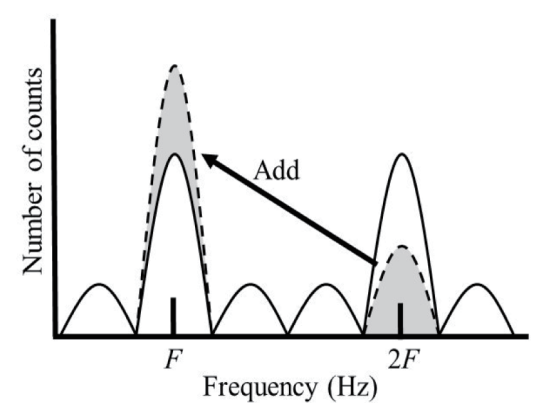

(b)

Fig. 4. Histograms (a) before and (b) after signal correction at close range. 
However, as the frequency of the second harmonic wave is $2 F$, the number of counts of the harmonic wave in the histogram is almost equal to that for the MHP. As a result, the harmonic wave may be erroneously detected as the MHP in the histogram. Therefore, processing is executed such that half of the number of counts of the reference frequency [ $2 F$ in Fig. 4(b)] is added to the number of counts of half of the reference frequency $[F$ in Fig. 4(b)]. Figure 4(b) is a schematic of the histogram obtained using this processing, which is carried out at close range. For a close-range measurement, even if the number of harmonic wave counts is equal to that of the MHP, the MHP is detected as the mode using this processing. Figure 5(a) is a schematic of the histogram for longrange measurement. The returned-light intensity is weak and the BPF suppresses the harmonic wave voltage. In addition, the number of counts of the low-frequency band is small. Thus, even if this addition process is executed for long-range measurement, the MHP frequency can be calculated as the mode value, as shown in Fig. 5(b).

\subsection{Measurement system}

A schematic of the measurement system is shown in Fig. 6, and an experimental photo is shown in Fig. 7. The LD is a ULM850-B2-PL-S46FZP (ULM850), with $850 \mathrm{~nm}$ wavelength, $2 \mathrm{~mW}$

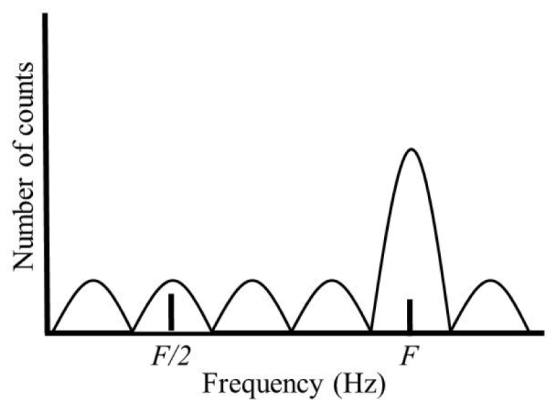

(a)

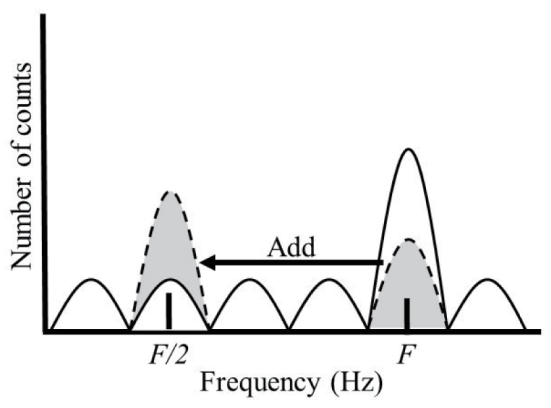

(b)

Fig. 5. Histograms (a) before and (b) after signal correction at long range.

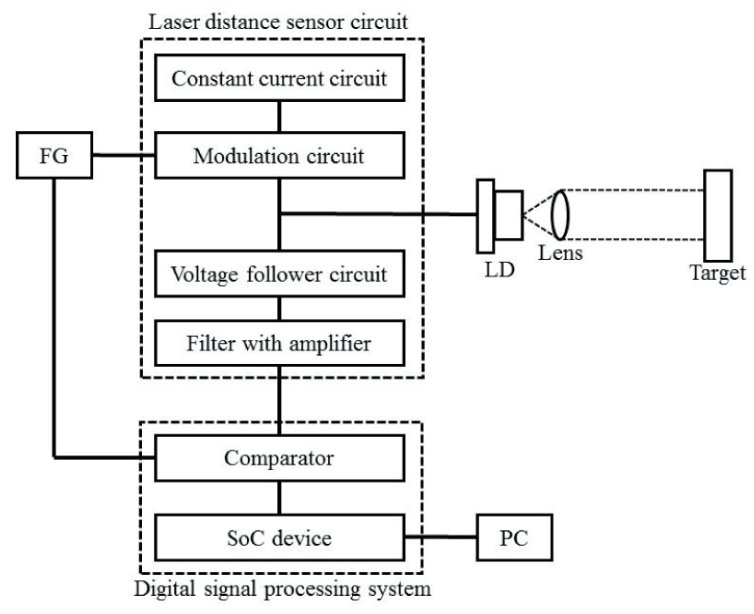

Fig. 6. Measurement system.

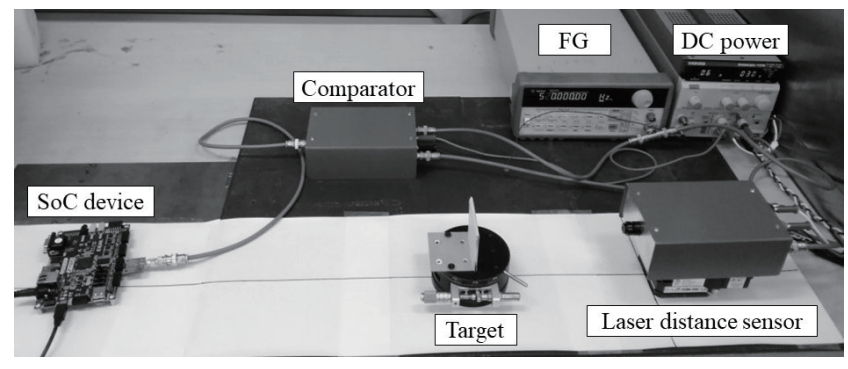

Fig. 7. Experimental photo. 
output-light intensity, single beam mode, and circular polarization. The driving circuit applies a $520 \mathrm{~Hz}$ triangular wave with a $1.8 \mathrm{~mA} \mathrm{p}-\mathrm{p}$ triangular current using a function generator. The ULM850 modulation efficiency is $0.46 \mathrm{~nm} / \mathrm{mA}$. The receiver is designed as a multistage BPF with 2500× amplification, with upper and lower cutoff frequency limits of $2 \mathrm{MHz}$ and $100 \mathrm{kHz}$, respectively. The laser beam is adjusted to yield a collimated beam and the target object is a white sheet. The SoC device is a Zybo made by Digilent. The total number of look-up tables (LUTs) is 17600 , the LUTs used after compilation are 828 , so the LUT utilization rate is $4 \%$, which is less than $1 / 10$ th of the LUT utilization rate of the digital circuit designed in the previous paper. ${ }^{(14)}$ Using the SoC device, signal processing for the histogram becomes easy and the LUT utilization rate is reduced. The data acquisition time is approximately $2 \mathrm{~ms}$, which is approximately equal to one period of the modulated signal. The delay-circuit delay time is $19.2 \mathrm{~ns}$; this circuit is synchronized with a clock signal of approximately $20 \mathrm{MHz}$. The pulse frequency for measuring the pulse width is $125 \mathrm{MHz}$. The time required for statistical addition processing is very short, and the overall processing time is almost identical to the data acquisition time. The measurement results are displayed on a personal computer.

\section{Results and Discussion}

Figure 8 shows graphs of the changes in the logic level at a target distance of $20 \mathrm{~cm}$ when noise reduction processing was performed. The sampling interval was $83 \mathrm{~ns}$ and the total number of sampled data points was 23974. The theoretical value of $F$ was 469 kHz. From Fig. 8(a), it is apparent that MHP selection is impossible. In contrast, Fig. 8(b) shows that a binarized high-level signal occurs periodically after noise reduction. This binarized high-level signal has $480 \mathrm{kHz}$ frequency, i.e., almost the theoretical value. Thus, it is confirmed that the reduction of signals other than the MHP is achievable.

Figure 9 is a histogram created for a $20 \mathrm{~cm}$ target distance after the execution of the entire process. A high intensity signal near the theoretical value can be confirmed. In addition, the intensity of the signal range equivalent to the second harmonic wave is also high. The detected signal may spread over a wide band similar to the second harmonic wave in Fig. 9, because the noise reduction processing and the signal measurement are performed simultaneously.

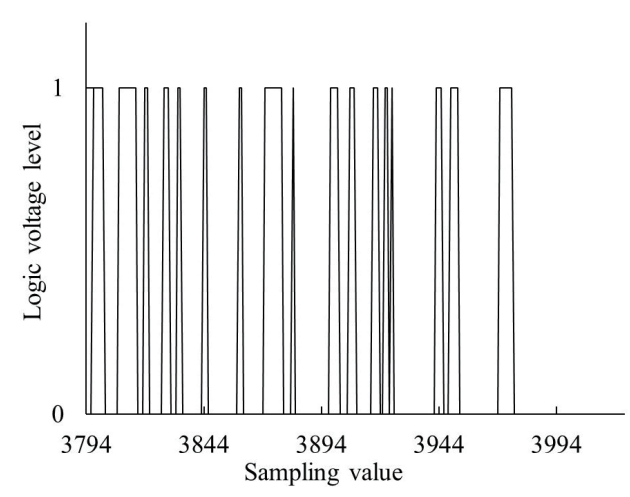

(a)

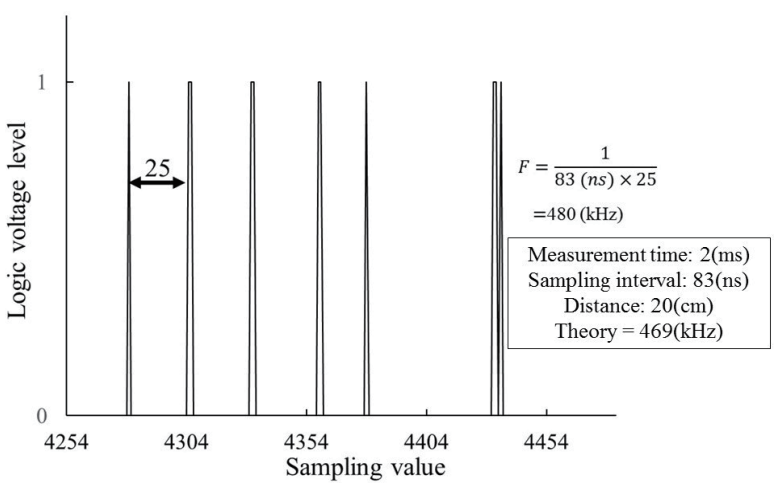

(b)

Fig. 8. Measured changes in logic level upon noise reduction processing. (a) Before and (b) after noise reduction. 


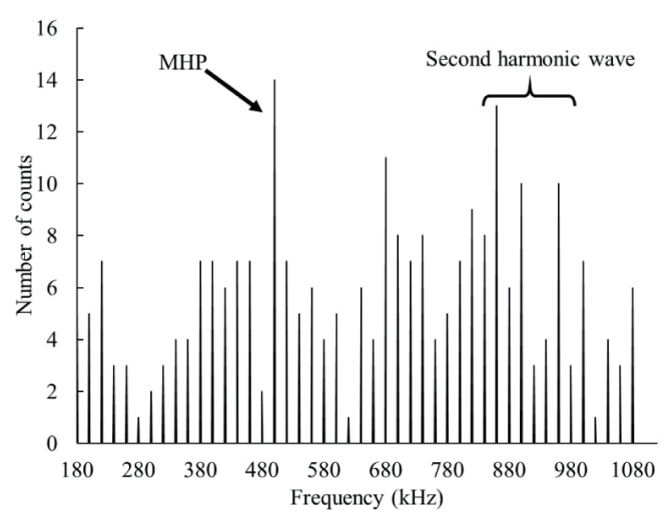

Fig. 9. Measured histogram for $20 \mathrm{~cm}$ target distance.

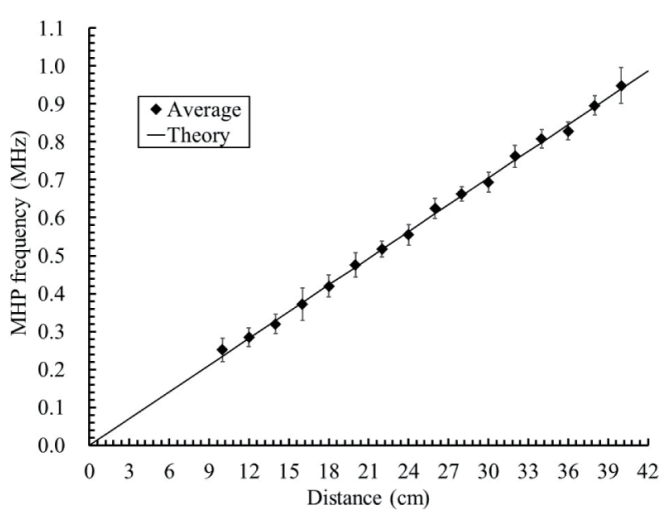

Fig. 10. Distance measurement results.

Measurement results for various target distances are shown in Fig. 10. The theoretical fit line was calculated using Eq. (1). Measurements were performed 20 times for each target distance and the average was calculated. The error bar in Fig. 10 represents the standard deviation calculated for each distance. It is confirmed that $F$ increases linearly with increased measurement distance. The maximum standard deviation is $0.047 \mathrm{MHz}$. In other words, the distance measurement value may vary by approximately $2 \mathrm{~cm}$. As the period of the MHP becomes longer for closer target distance, considerable noise is generated during one period of the binarized MHP. Thus, the time required for noise reduction is increased. In addition, as the MHP pulse width decreases with increasing distance, the difference between the MHP pulse width and the short-period-noise pulse width is also decreased. As a result, it is difficult to reduce the short-period noise. From the above solution, the real-time measurable range is determined. Compared with the measurement results of the previous report, ${ }^{(14)}$ the measurement time is shortened and the measurable range is increased. As the measurement accuracy yielded by the FFT is slightly better than that provided by the proposed method, further development to improve the accuracy of this method is necessary. On the other hand, the advantage of this measurement system is that the MHP is calculated from the output voltage, despite strong noise, via simple signal processing, and real-time measurement is possible.

\section{Conclusions}

We propose a new signal processing technique for the terminal voltage of a photodiode-free $\mathrm{LD}$, which allows distance measurement in real time. The terminal voltage of the photodiodefree LD includes the MHP, WGN, and the second harmonic wave of the MHP. The MHP is a periodic signal and the WGN is a random signal. Therefore, the increasing or decreasing interval of the triangular signal is divided. The MHP is detected by the repeated calculation of the logical product. The WGN within the LD terminal voltage is reduced using this method. In addition, in the histogram, half the number of high-frequency counts is added to the low-frequency counts. This processing resolves the problem in which the second harmonic wave is erroneously identified as the MHP. 
As a result, distance can be measured in one period of the modulated signal. Although the measurement accuracy achieved using FFT analysis is slightly better than that yielded by this method, the advantage of this measurement system is that the MHP is calculated via simple signal processing from the output voltage despite strong noise and, furthermore, real-time measurement is possible.

\section{Acknowledgments}

This work was supported by JSPS KAKENHI Grant Number (17K06470).

\section{References}

1 A. Sakamoto, N. Tsuda, and J. Yamada: IEEJ Trans. EIS-C 126 (2006) 1454 (in Japanese).

2 Y. Nakao, N. Tsuda, and J. Yamada: IEEJ Trans. EIS-C 121 (2001) 1819 (in Japanese).

3 T. Yoshimatsu, K. Goshima, N. Tsuda, and J. Yamada: IEEJ Trans. EIS-C 134 (2014) 1796 (in Japanese).

4 T. Gharbi, A. Courtevill, and A. Chebbour: Appl. Opt. 36 (1997) 8233.

5 G. Giuliani, S. Donati, and L. Monti: Proc. SPIE 4827 (2002) 353.

6 Y. Nawa, N. Tsuda, and J. Yamada: IEEJ Trans. EIS-C 129 (2009) 2115 (in Japanese).

7 P. J. Groot, G. M. Gallatin, and S. H. Macomber: Appl. Opt. 21 (1988) 4475.

8 L. Krehut, J. Hast, E. Alarousu, and R. Myllyla: Opto-Electron. Rev. 11 (2003) 313.

9 D. Mizushima, T. Yoshimatsu, K. Goshima, N. Tsuda, and J. Yamada: IEEJ Trans. EIS-C 136 (2015) 1021 (in Japanese).

10 D. Mizushima, T. Yoshimatsu, T. Yamaguchi, K. Goshima, N. Tsuda, and J. Yamada: IEEJ Trans. EIS-C 137 (2016) 489 (in Japanese).

11 C. Gorecki, S. Khalfallah, H. Kawakatsu, and Y. Arakawa: Sens. Actuators, A 87 (2001) 113.

12 J. Hashizume, S. Shinada, F. Koyama, and K. Iga: Opt. Rev. 9 (2002) 186.

13 T. Ueno, N. Tsuda, and J. Yamada: IEEJ Trans. EIS-C 130 (2010) 2100 (in Japanese).

14 T. Yoshimatsu, K. Goshima, M. Aoki, N. Tsuda, and J. Yamada: Rev. Laser Eng. 44 (2016) 679 (in Japanese).

15 T. Yoshimatsu, N. Tsuda, and J. Yamada: Proc. 10th Int. Conf. Sensing Technology (ICST 2016) p. 497.

16 S. Shinohara, A. Mochizuki, H. Yoshida, and M. Sumi: Appl. Opt. 25 (1986) 1416.

\section{About the Authors}

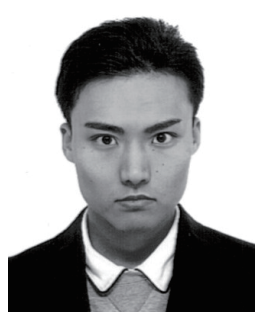

Takeshi Yoshimatsu received his M.E. degree from Aichi Institute of Technology (AIT). He is currently studying at the Department of Electricity and Materials Engineering, AIT. His research interests include laser measurement.

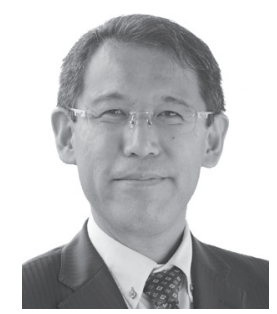

Norio Tsuda received his Ph.D. degree from AIT. He now works with the Department of Electronics and Electrical Engineering, AIT. His research interests include the physical properties of high-pressure laser-induced plasmas and laser measurements using the self-coupling effect of semiconductor lasers. He is a member of the Laser Society of Japan, the Physical Society of Japan, and the Institute of Electrical Engineers of Japan. 


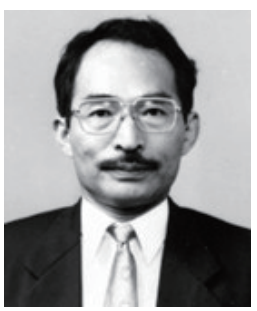

Jun Yamada received his Ph.D. degree from Nagoya University. He is now an emeritus professor at AIT. His research interests include the physical properties of high-pressure laser-induced plasmas and laser measurements using the selfcoupling effect of semiconductor lasers. He is a member of the Laser Society of Japan and the Institute of Electrical Engineers of Japan. 